\title{
A Comparative Study on the Effects of
} Postoperative ${ }^{125}$ I Brachytherapy and Irradiation After Surgical Decompression and Stabilization
for Metastatic Spinal Cancers

This article was published in the following Dove Press journal: Journal of Multidisciplinary Healthcare

\section{Xuedong $\mathrm{Shi}^{1}{ }^{1} *$ \\ Yaosheng $\mathrm{Liu}^{2} *$ \\ Yunpeng Cui \\ Haifeng Qin (1D ${ }^{3}$ \\ Shaoxing Yang ${ }^{3}$ \\ Mingxing Lei ${ }^{4}$}

'Department of Orthopedic Surgery, Peking University First Hospital, Beijing, People's Republic of China; ${ }^{2}$ Department of Orthopedic Surgery, The Fourth Medical Center of Chinese PLA General Hospital, Beijing, People's Republic of China; ${ }^{3}$ Department of Pulmonary Neoplasms Internal Medicine, The Fifth Medical Center of the Chinese PLA General Hospital, Beijing, People's Republic of China; ${ }^{4}$ Department of Orthopedic Surgery, Hainan Hospital of Chinese PLA General Hospital, Sanya, People's Republic of China

*These authors contributed equally to this work

\begin{abstract}
Purpose: The study aims to investigate and compare the efficacy and safety of intraoperative ${ }^{125} \mathrm{I}$ implantation and postoperative irradiation after surgical decompression and stabilization in the treatment of patients with metastatic epidural spinal cord compression (MESCC).
\end{abstract}

Methods: The study retrospectively enrolled 122 MESCC patients treated with surgical decompression and pedicle stabilization combined with ${ }^{125} \mathrm{I}$ brachytherapy (the brachytherapy group) or postoperative radiotherapy (the irradiation group). Operation time, intraoperative blood loss, pain relief, postoperative ambulatory status, postoperative survival outcome, complications, and length of hospitalization were collected and compared between the two groups. Ten potential risk factors were analyzed for postoperative survival outcome.

Results: No significant difference was found in baseline characteristics between the two groups $(\mathrm{P}>0.05)$. Postoperative VAS score was significantly decreased, as compared with preoperative scores in both groups $(\mathrm{P}<0.001)$. The VAS in the brachytherapy group was significantly lower than that in the irradiation group at postoperative $1 \mathrm{month}, 3$ months, and 6 months $(\mathrm{P}<0.05)$. The postoperative ambulatory rates were $90.0 \%(54 / 60)$ in the brachytherapy group and $83.9 \%(52 / 62)$ in the irradiation group $(\mathrm{P}=0.32)$. The median overall survival time was similar between the two groups ( 7.43 months vs 7.27 months, $\mathrm{P}=0.37$ ). Of all patients in the brachytherapy group, $25.0 \%$ (15/60) of patients suffered from complications, while $46.8 \%(29 / 62)$ of patients had complications in the irradiation group $(\mathrm{P}=0.0086)$. According to the multiple Cox regression, primary sites $(\mathrm{P}=0.038)$, ECOG performance status $(\mathrm{P}=0.014)$, and visceral metastases $(\mathrm{P}=0.0016)$ showed significance for postoperative survival outcome.

Conclusion: Surgical decompression and spine stabilization combined with ${ }^{125}$ I brachytherapy is a relatively safe and useful method in MESCC patients.

Keywords: metastatic epidural spinal cord compression, ${ }^{125} \mathrm{I}$ brachytherapy, radiotherapy, surgical decompression and spine stabilization, prognosis

\section{Introduction}

Metastatic epidural spinal cord compression (MESCC), a common complication of malignant tumors, occurs when malignant tumors metastasize to the vertebra or epidural space and consequently causes spinal cord compression. ${ }^{1,2}$ MESCC requires interdisciplinary cooperation, ${ }^{1,2}$ including decompressive surgery, minimally invasive
Department of Orthopedic Surgery, The Fourth Medical Center of Chinese PLA

General Hospital, No. 8, Fengtaidongda

Road, Beijing 10007I, People's Republic of

China

Tel/Fax +86-10-66947017

Email 632763246@qq.com 
technique, radiotherapy, embolization, and systematic internal medicine. Recently, increasing researchers revealed that direct decompressive and stabilized surgery followed by radiotherapy was superior to radiotherapy alone in terms of postoperative ambulatory status, pain relief, and even survival prognosis. ${ }^{3-5}$ Therefore, surgical decompression and spine stabilization followed by postoperative radiotherapy has become increasing popular for MESCC patients.

However, patients treated with radiation had plenty of acute or subacute toxicities, subsequently requiring additional interventions, impacting patient's quality of life, and burdening medical costs. Radiation therapy was known to impair wound healing regardless of the fractionation of radiation. ${ }^{6}$ It was reported that $53.9 \%$ to $63.0 \%$ radiationtreated patients with spine metastasis suffered from toxicities, ${ }^{7-9}$ such as gastrointestinal toxicity and even spinal cord damage. Although stereotactic body radiation therapy could provide better pain relief and local control of disease with a relatively low toxicity rate $(32.1 \%$ to $38.5 \%)$, it was not widely used due to high total gross charge. ${ }^{7-9}$

${ }^{125}$ I seed implantation is a booming method which yields encouraging clinical results in the treatment of spine metastasis. Percutaneous vertebroplasty (PVP) combined with ${ }^{125} \mathrm{I}$ implantation was superior to radiotherapy alone in terms of pain relief and patient's quality of life in patients with spine metastasis. ${ }^{10,11}$ Decompressive surgery combining with ${ }^{125}$ I brachytherapy seed implantation facilitates rapid decompression, immediate metastatic radiation, and accurate tumor assessment, which has become a new modality for MESCC patients. ${ }^{12,13}$ However, the available studies were subject to small and retrospective samples, simple observations, and indistinct endpoints. To our knowledge, there is no report comparing the efficacy and safety of surgical decompression and spine stabilization combined with ${ }^{125}$ I brachytherapy and with postoperative radiotherapy in the treatment of MESCC patients.

Therefore, we speculated ${ }^{125} \mathrm{I}$ brachytherapy could obtain better pain relief and less radiation-related complications. The study aims to investigate and compare the efficacy and safety of surgical decompression and spine stabilization combined with ${ }^{125} \mathrm{I}$ brachytherapy and postoperative radiotherapy in MESCC patients.

\section{Methods}

\section{Patients}

We retrospectively enrolled 122 patients with MESCC admitted to our departments between January 2017 and
December 2019. Patients were included if they had (1) an age of more than 18 years, (2) MRI evidence of MESCC, (3) clear diagnosis of malignant tumor, (4) severe back pain or neurological deficit due to MESCC, and (5) treated with surgical decompression and pedicle stabilization combined with ${ }^{125}$ I brachytherapy or combined with postoperative radiotherapy. Patients were excluded if they had (1) intradural metastases, (2) vertebrae metastatic lesions previously treated by surgery or radiotherapy, (3) pathological fracture in the lower limbs, (4) too poor health to undergo surgery, and (5) paralysis more than 42 hours. A study profile is shown in Figure 1. Patients were divided into two groups: patients in the brachytherapy group were treated with surgical decompression and pedicle stabilization combined with ${ }^{125} \mathrm{I}$ brachytherapy; patients in the postoperative irradiation group were treated with surgical decompression and pedicle stabilization combined with postoperative radiotherapy. The study was approved by the Medical Research Ethics Board of the Fifth Medical Center of Chinese PLA General Hospital. Follow-up records were obtained with the patient's informed consent. The study was conducted following the Declaration of Helsinki.

\section{Procedures}

Patients in the brachytherapy group were treated with surgical decompression and pedicle stabilization combined with

${ }^{125}$ I brachytherapy. Patient's involved vertebrae were performed with a 64-slice CT scan before surgery. The scan thickness was $0.625 \mathrm{~mm}$. The images were imported into $3 \mathrm{D}$ treatment planning system (3D-TPS). The dose of ${ }^{125} \mathrm{I}$ seeds was calculated according to the shape and size of lesions. The size of ${ }^{125} \mathrm{I}$ is $0.8 \mathrm{~mm} \times 4.2 \mathrm{~mm}$, which is contained by titanium alloy, the half-life is 60.2 days, and the activity is $0.6 \sim 0.9 \mathrm{mCi}$ per seed. Besides, the images of CT were saved in DICOM format. These files were imported into MIMICS 10.01 software (Materialise Company, Belgium) to rebuild the 3D model which was then exported as STL format. UG Imageware 12.0 (EDS. USA) was used to open the $3 \mathrm{D}$ model and design the appropriate position and direction of seeds insertion into the involved vertebra.

After the successful induction of general anesthesia, intubation was inserted with the help of a laryngoscope. The patient was placed in the prone position, routinely disinfected and draped in a sterile manner. G-arm fluoroscopy was used to locate the involved vertebra. Incisions were made in the center of the involved vertebra, and then the subcutaneous tissue and fascia were stripped off. The 


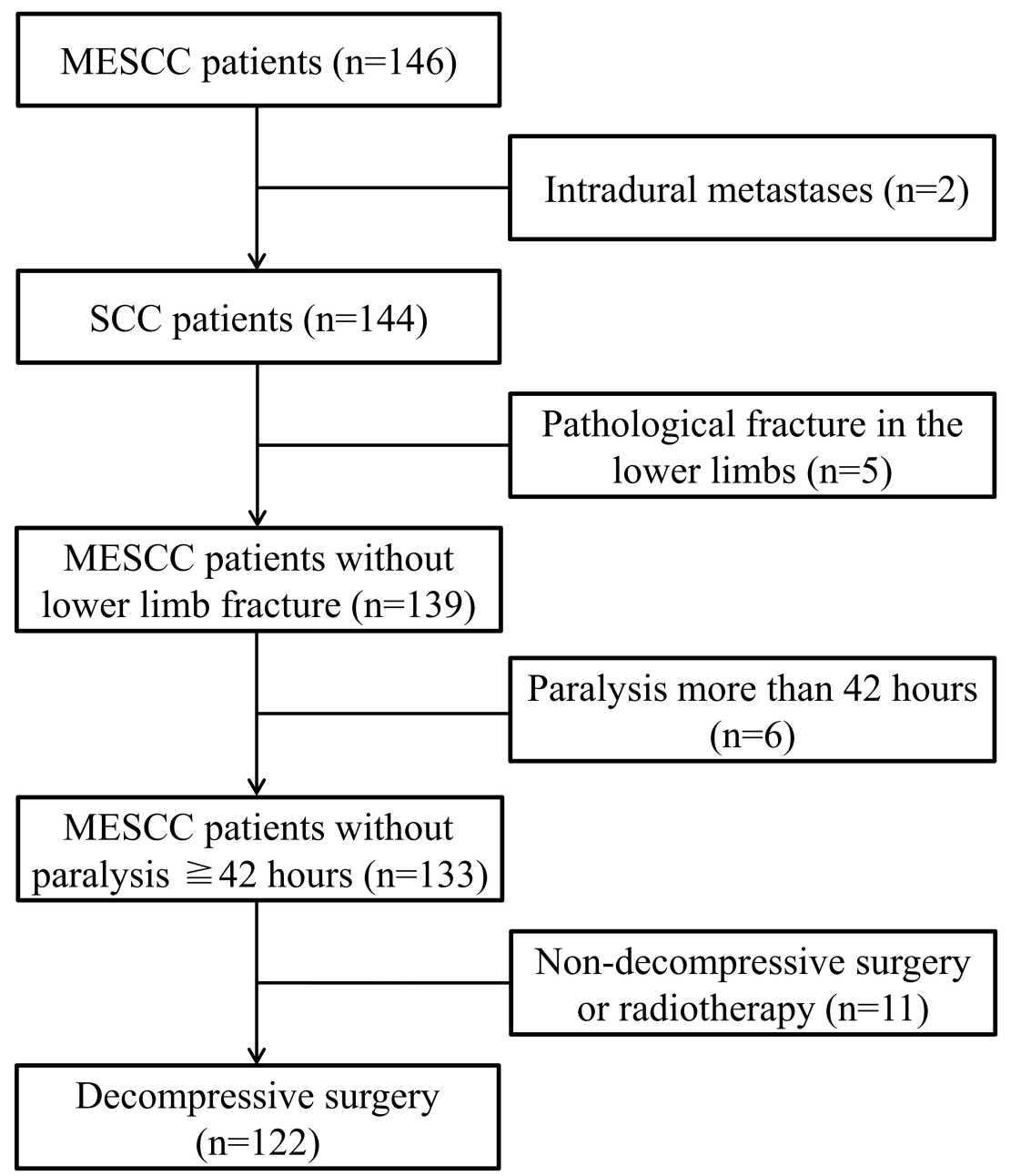

Figure I The study profile. SCC indicates spinal cord compression; MESCC indicates metastatic epidural spinal cord compression.

lamina of the involved vertebra was exposed using a detacher. After the pedicles of the adjacent noninvolved vertebras were placed with pedicle screws, a spinous rongeur was used to bite off the spinous process and the surrounding soft tissue, and a lamina rongeur was used to remove the lamina of the involved vertebra. Palliative tumor resection of the intra-vertebra was performed. Surgeons inserted two $18 \mathrm{G}$ puncture needles into the front of the involved vertebrae. G-arm fluoroscopy was used to confirm the guide needle was in an ideal position. The radioactive ${ }^{125}$ I seeds were placed in the gun and were gradually implanted into the metastatic lesions. Finally, the G-arm fluoroscopy was performed to confirm that both pedicle screws and radioactive ${ }^{125}$ I seeds were in suitable positions. A drainage tube was placed under the incision. After the installation of the connecting rod and fixation of the end caps, the incision was sutured layer by layer. Figures 2 and 3 show a case report.
For patients in the irradiation group, surgical decompression and spine stabilization were performed within 48 hours after randomization. Apart from the implantation of radioactive ${ }^{125}$ I seeds, the other procedures were similar to the brachytherapy group. Conventional radiation was administrated after wound healing, about 2 to 4 weeks after surgery, and the dose was 8 Gray in a single fraction.

\section{Outcome Assessments}

Patient's baseline characteristics, including age, gender, primary cancer sites, preoperative ambulatory status, Eastern Cooperative Oncology Group (ECOG) performance status, visceral metastases, bone metastasis at cancer diagnosis, extra-spinal bone metastases, and time developing motor deficits, were recorded and compared between the two groups. Operation time, intraoperative blood loss, pain relief, postoperative ambulatory status, 

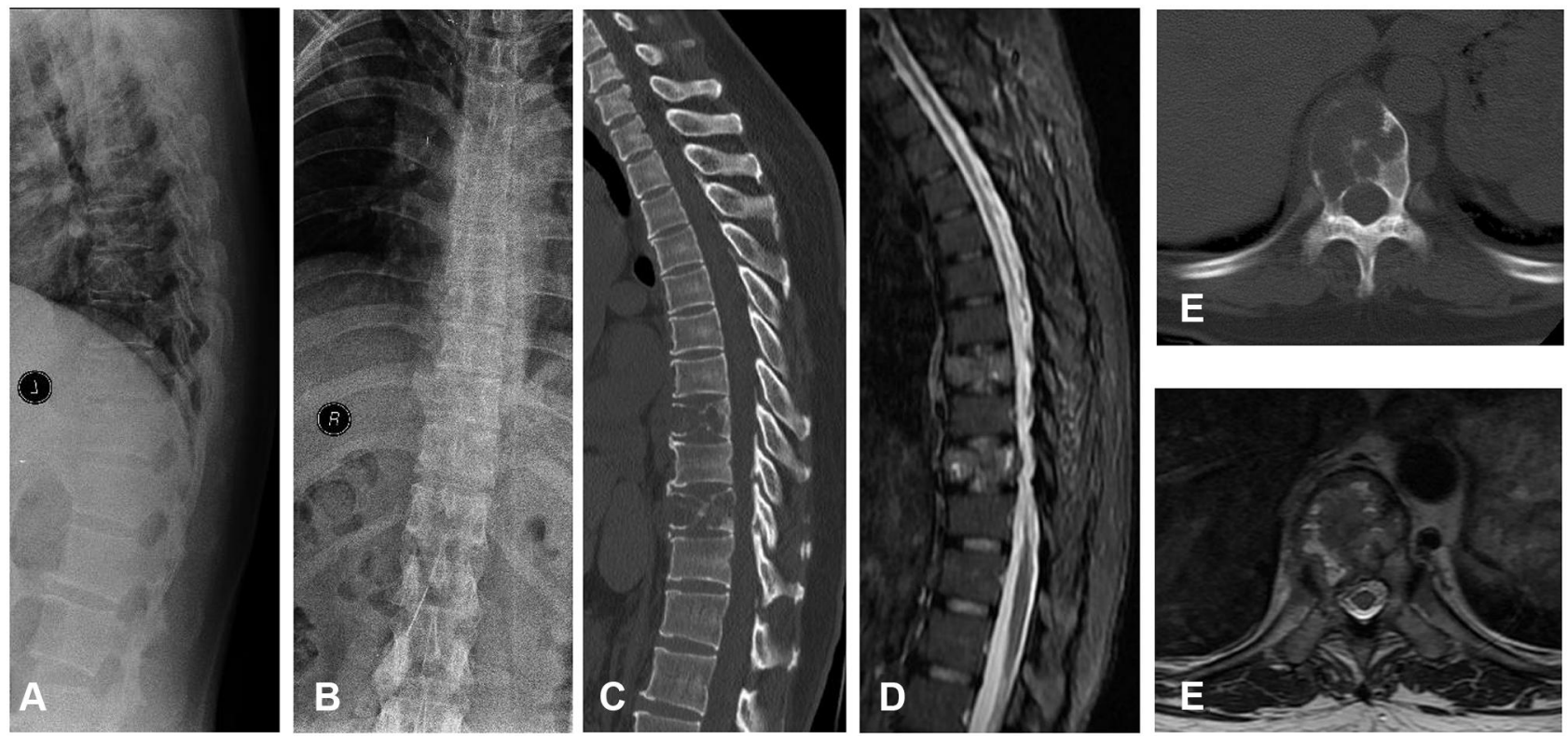

Figure 2 A 47-year-old female diagnosed with lung cancer treated with surgical decompression and pedicle stabilization combined with ${ }^{125}$ I brachytherapy seeds implantation. (A) Preoperative lateral X-ray showed T6 and T8 vertebral collapse. (B) Preoperative anteroposterior X-ray showed T6 and T8 vertebral collapse. (C) Preoperative lateral CT showed T6 and T8 bone destruction. (D) Preoperative lateral MRI showed T6 and T8 spine metastases. (E) Preoperative transverse CT showed bone destruction at T8. (F) Preoperative transverse MRI showed deformation of the dural sac due to metastatic tumor.
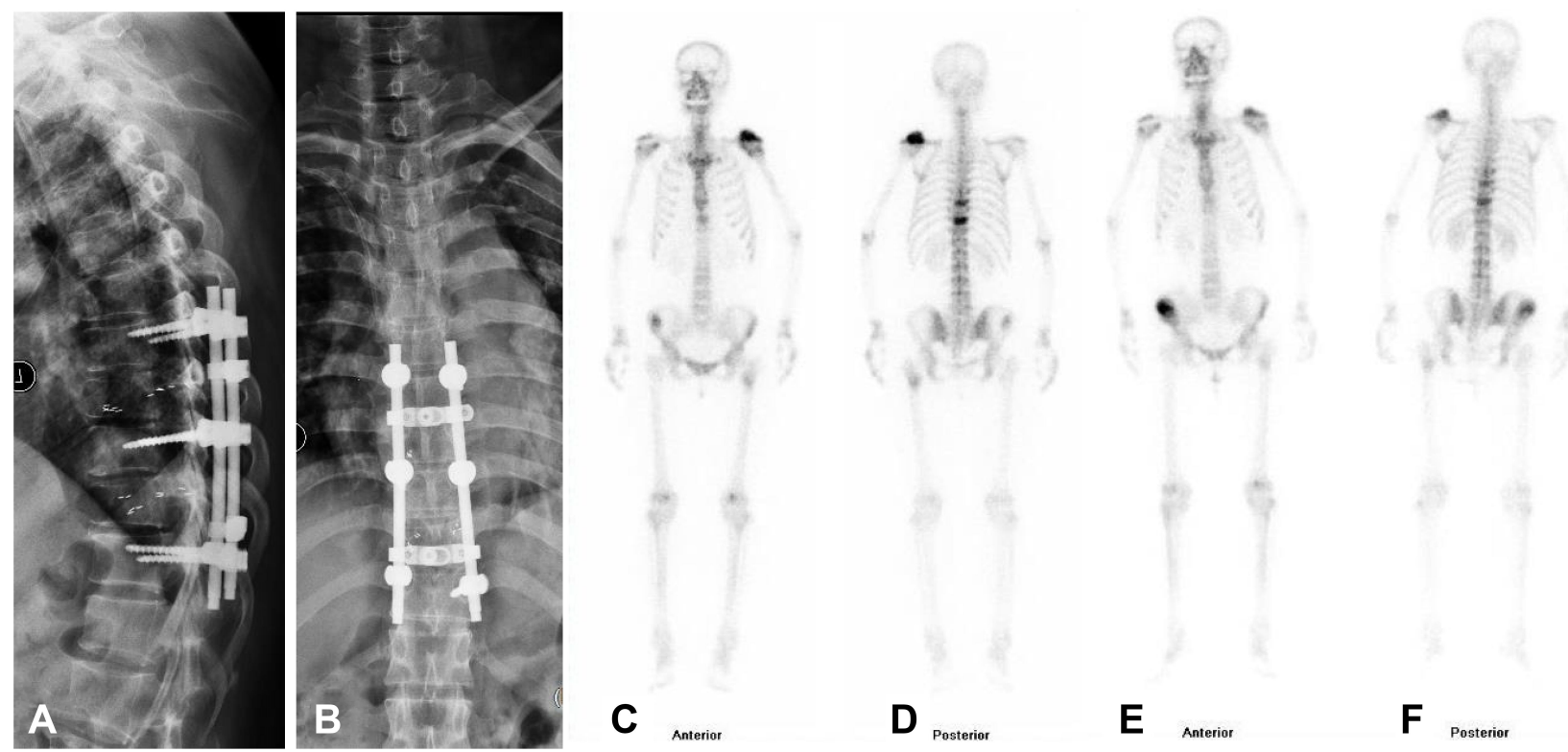

Figure 3 (A) Postoperative lateral X-ray showed ${ }^{125}$ I brachytherapy seeds implantation and pedicle stabilization with screws. (B) Postoperative anteroposterior $X$-ray showed ${ }^{125} \mathrm{I}$ brachytherapy seeds implantation and pedicle stabilization with screws. (C) Anterior bone scan at postoperative 10 months. (D) Posterior bone scan at postoperative 19 months. (E) Anterior bone scan at postoperative 19 months. (F) Posterior bone scan at postoperative 19 months. The above bone scan indicated no disease progression at 19 months after surgery.

postoperative survival time, length of hospitalization, and complications were analyzed between the two groups.

Operation time was defined as the time interval between skin incision and suture. Intraoperative blood loss was recorded during surgery. Pain relief was evaluated using Visual analogue score (VAS). VAS ranged from 0 representing no pain to 10 representing the maximum pain in life. VAS was collected before surgery and at the time of discharge, 1 month, 3 months, and 6 months after surgery. Postoperative ambulatory status was evaluated using Frankel scores before surgery and 4 weeks after surgery. In detail, Frankel A to $\mathrm{C}$ was non-ambulatory, 
while Frankel D and E were ambulatory. If a patient could ambulate with or without aid, the patient was regarded as having the ability to walk. ${ }^{3}$ Survival time was defined as the time interval between surgery and death data or the last follow up. Patients who were alive at the last follow-up were censored in the overall survival analysis. Length of hospitalization was defined as the time interval between the data of hospitalization and discharge. Complications were classified into surgery-related complications, such as operation site infection, wound dehiscence, cerebrospinal fluid leakage, and epidural hematoma, radiation-related complications, such as abdominal complaints (diarrhea, abdominal pain, nausea, and vomiting) and skin complaints (itching and painful skin), and systematic complications, such as pneumonia, pulmonary embolism, stroke, septicemia, intestinal bleeding, and multiple organ failure.

\section{Screening Predictors for Survival Outcome}

The Cox regression model was used to screen the ten potential risk factors for postoperative survival outcome. The ten factors included age (years), gender (female vs male), primary cancer sites (slow growth vs moderate growth vs rapid growth, conformed to previous studies ${ }^{14}$ ), preoperative ambulatory status (ambulatory vs not ambulatory), ECOG performance status (1-2 vs 3-4), visceral metastases (No vs Yes), bone metastasis at cancer diagnosis (No vs Yes), extra-spinal bone metastases (No vs Yes), time developing motor deficits ( $\leq 14$ days vs $>14$ days, conformed to previous studies ${ }^{14}$ ), and treatment strategies (the brachytherapy group vs the irradiation group).

\section{Statistical Analysis}

The Chi-square test and $t$-test were performed to analyze the baseline characteristics between the brachytherapy and irradiation group. Analysis of the VAS between the two groups was performed via repeated measures of the correlated variance model, supplemented by Wilcoxon ranksum test. Postoperative ambulatory status between the two groups was compared by the Chi-square test. Postoperative overall survival prognosis was evaluated using the Kaplan-Meier method and Log rank test. Complications were assessed by the Chi-square test and the hospitalization length was measured by Wilcoxon rank test. The univariate and multivariate analyses of postoperative survival outcome were estimated by the simple and multiple Cox proportional hazards regression models, respectively. A $\mathrm{P}$ value of 0.05 or less was considered statistically significant. Statistical analysis was performed using SAS 9.2 software.

\section{Results \\ Patient's Characteristics}

The brachytherapy group included 60 patients and the irradiation group had 62 patients. The mean age was $58.3 \pm 11.0$ years old in the brachytherapy group and $57.3 \pm 14.4$ years old in the irradiation group $(\mathrm{P}=0.66$, Table 1$)$. There were 31 female patients in the brachytherapy group and 34 female patients in the irradiation group $(\mathrm{P}=0.73)$. The majority of cancer type was rapid growth cancer, which accounted for $51.7 \%(31 / 60)$ in the brachytherapy group and $51.6 \%(32 / 62)$ in the irradiation group ( $\mathrm{P}=0.13)$. In detail, 27 patients had lung cancer, 15 patients had breast cancer, 4 patients had liver cancer, 3 patients had prostate cancer, and 11 patients had other cancers in the brachytherapy group. In the irradiation group, 28 patients had lung cancer, 14 patients had breast cancer, 4 patients had liver cancer, 6 patients had prostate cancer, and 10 patients had other cancers. Most of the patients had unsatisfactory performance status in both groups $(\mathrm{P}=0.25)$. The distribution of visceral metastases, bone metastasis at cancer diagnosis, and extra-spinal bone metastases were also similar between the two groups $(\mathrm{P}>0.05)$.

\section{Pain Relief}

The mean pain score in 24-hour period was $7.93 \pm 1.44$ in the brachytherapy group before surgery. At the time of discharge, one month, three months, and six months after the operation, mean scores decreased to $2.72 \pm 1.12(\mathrm{P}<0.001), 1.75 \pm 0.78$ $(\mathrm{P}<0.001), 2.35 \pm 0.83(\mathrm{P}<0.001)$, and 3.39 $\pm 1.00(\mathrm{P}<0.001)$, respectively (Table 2). Similar decreases in VAS were observed in the irradiation group. The mean pain score was $7.69 \pm 1.13$ in the irradiation group before surgery, and the mean scores decreased to $3.02 \pm 1.50(\mathrm{P}<0.001)$ at the time of discharge, 2.95 $\pm 1.31 \quad(\mathrm{P}<0.001)$ at 1 month, $3.53 \pm 1.77$ $(\mathrm{P}<0.001)$ at three months, and $4.00 \pm 1.42$ at six months $(\mathrm{P}<0.001)$ after surgery. VAS was similar between the two groups at the time of discharge $(2.72 \pm 1.12$ vs $3.02 \pm 1.50$, $\mathrm{P}=0.20$ ). However, VAS in the brachytherapy group was significantly lower than that in the irradiation group one month, three months, and six months after surgery $(\mathrm{P}<0.05$, Figure 4$)$.

\section{Function Outcome}

The postoperative ambulatory rates were $90.0 \%(54 / 60)$ in the brachytherapy group and $83.9 \%$ (52/62) in the 
Table I Patient's Characteristics of the Brachytherapy and Irradiation Group

\begin{tabular}{|c|c|c|c|}
\hline Characteristics & The Brachytherapy Group $(n=60)$ & The Irradiation Group $(n=62)$ & P-values \\
\hline Age (mean, years) & $58.3 \pm 11.0$ & $57.3 \pm 14.4$ & 0.66 \\
\hline \multicolumn{4}{|l|}{ Gender } \\
\hline Female & 31 & 34 & 0.73 \\
\hline Male & 29 & 28 & \\
\hline \multicolumn{4}{|l|}{ Primary cancer sites } \\
\hline Slow growth & 15 & 7 & 0.13 \\
\hline Moderate growth & 14 & 23 & \\
\hline Rapid growth & 31 & 32 & \\
\hline \multicolumn{4}{|l|}{ Preoperative ambulatory status } \\
\hline Ambulatory & 42 & 39 & 0.41 \\
\hline Not Ambulatory & 18 & 23 & \\
\hline \multicolumn{4}{|l|}{ ECOG performance status } \\
\hline $\mathrm{I}-2$ & 21 & 28 & 0.25 \\
\hline $3-4$ & 39 & 34 & \\
\hline \multicolumn{4}{|l|}{ Visceral metastases } \\
\hline No & 37 & 29 & 0.10 \\
\hline Yes & 23 & 33 & \\
\hline \multicolumn{4}{|l|}{ Bone metastasis at cancer diagnosis } \\
\hline No & 33 & 33 & 0.84 \\
\hline Yes & 27 & 29 & \\
\hline \multicolumn{4}{|l|}{ Extraspinal bone metastases } \\
\hline No & 33 & 34 & 0.99 \\
\hline Yes & 27 & 28 & \\
\hline \multicolumn{4}{|l|}{ Time developing motor deficits } \\
\hline$\leqq 14$ days & 31 & 30 & 0.72 \\
\hline$>14$ days & 29 & 32 & \\
\hline
\end{tabular}

Abbreviations: ECOG, Eastern Cooperative Oncology Group; VAS, visual analogue score; MESCC, metastatic epidural spinal cord compression.

irradiation group ( $\mathrm{P}=0.32$, Table 2$)$. In detail, in the brachytherapy group, $95.2 \%$ (40/42) patients who had the ability to walk before surgery sustained ambulation after surgery, and $92.3 \%$ (36/39) patients continued to ambulate in the irradiation group. Regarding patients who were unable to walk before surgery, $77.8 \%(14 / 18)$ of patients regained ambulation in the brachytherapy group and $69.6 \%(16 / 23)$ of patients in the irradiation group.

\section{Complications and Hospitalization}

Of all the patients in the brachytherapy group, 25.0\% (15/ 60) suffered from complications and $46.8 \%(29 / 62)$ patients had complications in the irradiation group $(\mathrm{P}=0.0086$, Table 2). In detail, patients in the brachytherapy group had a lower rate of radiation-related complications as compared with patients in the irradiation group $(8.3 \%$ vs $38.7 \%, \mathrm{P}<0.001)$. The rates of surgery-related
$(\mathrm{P}=0.35)$ and systematic complications $(\mathrm{P}=0.82)$ were similar between the two groups. In the brachytherapy group, eight patients had surgery-related complications, five patients had radiation-related complications, and six patients had systematic complications. In detail, three patients with surgery-related complications also had radiation-related complications. One patient with surgeryrelated complication also had systematic complication. In the irradiation group, five patients had surgery-related complications, 24 patients had radiation-related complications, and seven patients had systematic complications. In detail, three patients with surgery-related complications also had radiation-related complications, four patients with radiation-related complications also had systematic complications, and two patients with surgery-related complications also had systematic complications. Five patients received radiotherapy three to eight months after surgery 
Table 2 The Comparison of Intraoperative Variables and Postoperative Clinical Outcomes Between the Brachytherapy and Irradiation Group

\begin{tabular}{|c|c|c|c|}
\hline \multirow[t]{2}{*}{ Characteristics } & \multirow{2}{*}{$\begin{array}{l}\text { The Brachytherapy Group } \\
\text { Patients }(n=60)\end{array}$} & \multirow{2}{*}{$\begin{array}{l}\text { The Irradiation Group } \\
\text { Patients }(n=62)\end{array}$} & \multirow[t]{2}{*}{ P-values } \\
\hline & & & \\
\hline Operation time (mean, min) & $206 \pm 36$ & $190 \pm 34$ & 0.017 \\
\hline Intraoperative blood loss (mean, mL) & $562 \pm 117$ & $420 \pm 94$ & $<0.001$ \\
\hline Preoperative VAS (mean) & $7.93 \pm 1.44^{\mathrm{a}}$ & $7.69 \pm 1.13^{b}$ & 0.32 \\
\hline Postoperative VAS at the time of discharge & $2.72 \pm 1.12^{\mathrm{a}}$ & $3.02 \pm 1.50^{\mathrm{b}}$ & 0.20 \\
\hline Postoperative VAS at I months & $1.75 \pm 0.78^{\mathrm{a}}$ & $2.95 \pm 1.31^{\mathrm{b}}$ & $<0.00$ I \\
\hline Postoperative VAS at 3 months & $2.35 \pm 0.83^{\mathrm{a}}$ & $3.53 \pm 1.77^{b}$ & $<0.001$ \\
\hline Postoperative VAS at 6 months & $3.39 \pm 1.00^{\mathrm{a}}$ & $4.00 \pm 1.42^{b}$ & 0.039 \\
\hline \multicolumn{4}{|l|}{ Postoperative ambulatory status } \\
\hline Ambulatory & 54 & 52 & 0.32 \\
\hline Not ambulatory & 6 & 10 & \\
\hline Postoperative survival time (median, months) & 7.27 & 7.43 & 0.37 \\
\hline \multicolumn{4}{|l|}{ Complications } \\
\hline Yes & 15 & 29 & 0.0086 \\
\hline No & 47 & 33 & \\
\hline \multicolumn{4}{|l|}{ Surgery-related complications } \\
\hline Yes & 8 & 5 & 0.35 \\
\hline No & 52 & 57 & \\
\hline \multicolumn{4}{|l|}{ Radiation-related complications } \\
\hline Yes & 5 & 24 & $<0.00$ I \\
\hline No & 55 & 38 & \\
\hline \multicolumn{4}{|l|}{ Systematic complications } \\
\hline Yes & 6 & 7 & 0.82 \\
\hline No & 54 & 55 & \\
\hline The length of hospitalization (mean, days) & $21.2 \pm 5.8$ & $19.7 \pm 6.2$ & 0.17 \\
\hline
\end{tabular}

Notes: VAS, visual analogue score; ${ }^{a}$ All $\mathrm{P}<0.00 \mathrm{I}$, as compared with the preoperative VAS in the brachytherapy group; ${ }^{\mathrm{b}} \mathrm{All} \mathrm{P}<0.00 \mathrm{I}$, as compared with the preoperative VAS in the irradiation group.

due to disease progression, and radiotherapy-related complication occurred in one patient. Regarding hospitalization, the mean length was $21.2 \pm 5.8$ days in the brachytherapy group and $19.7 \pm 6.2$ days in the irradiation group $(\mathrm{P}=0.17)$.

\section{Survival Prognosis}

The median overall survival time was 7.27 months $(95 \%$ CI: 5.70-8.20 months) in the brachytherapy group and 7.43 months (95\% CI: 6.43-10.73 months) in the irradiation group ( $\mathrm{P}=0.37$, Log rank test, Figure 5$)$. In the brachytherapy group, the 6 and 12 months overall survival rates were $60.7 \%$ and $24.6 \%$, respectively. In the irradiation group, the 6 and 12 months overall survival rates were $65.2 \%$ and $29.7 \%$, respectively.

In the simple analysis of the ten factors, primary cancer sites $(\mathrm{HR}=1.55 ; 95 \% \mathrm{CI}: 1.19-2.03, \mathrm{P}=0.001), \mathrm{ECOG}$ performance status $\quad(\mathrm{HR}=2.19 ; \quad 95 \% \quad \mathrm{CI}: \quad 1.43-3.34$, $\mathrm{P}<0.001)$, and visceral metastases $(\mathrm{HR}=1.73$; 95\% CI: $1.17-2.55, \mathrm{P}=0.006)$ were significantly associated with postoperative overall survival time, but other seven factors were not significant (Table 3). According to the multiple Cox regression analysis, primary sites $(\mathrm{HR}=1.38$; $95 \% \mathrm{CI}$ : 1.02-1.87, $\mathrm{P}=0.038), \quad \mathrm{ECOG}$ performance status $(\mathrm{HR}=1.82 ; 95 \% \mathrm{CI}: 1.13-2.93, \mathrm{P}=0.014)$, and visceral metastases $(\mathrm{HR}=1.89 ;$ 95\% CI: 1.27-2.79, $\mathrm{P}=0.0016)$ maintained significance.

\section{Discussion}

In the present study, we retrospectively analyzed 146 MESCC patients and enrolled 122 patients. Of all the included patients, 60 patients were treated with surgical decompression and pedicle stabilization combined with ${ }^{125} \mathrm{I}$ brachytherapy and 62 were treated with surgical 


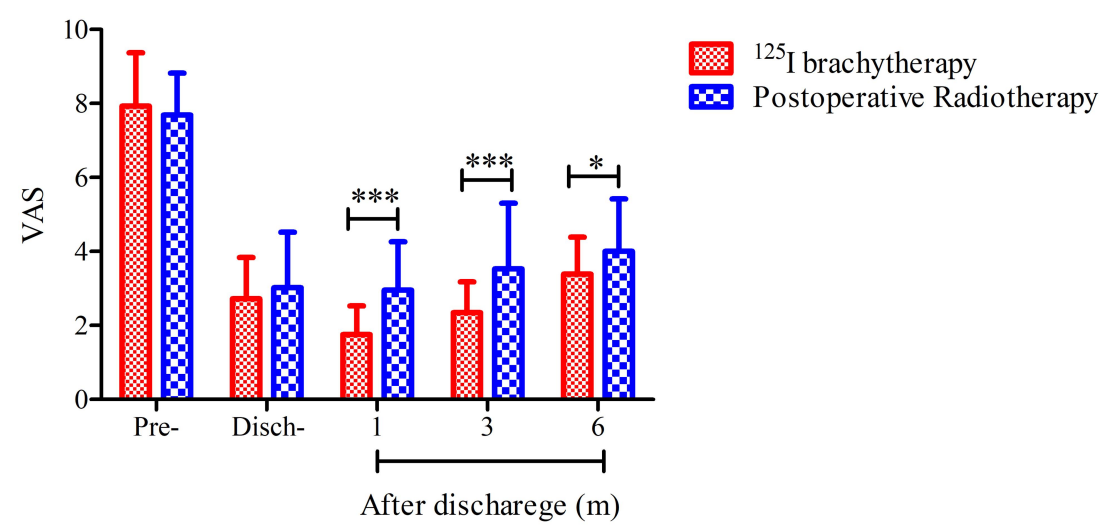

Figure 4 The mean VAS score in a 24-hour period before surgery and at the time of discharge, I month, three months, and six months after surgery in both groups. "pre-" indicates pre-operation; "disch-" indicates discharge; " $m$ " indicates months; *Indicates $P<0.05$; ***Indicates $P<0.0$ I.

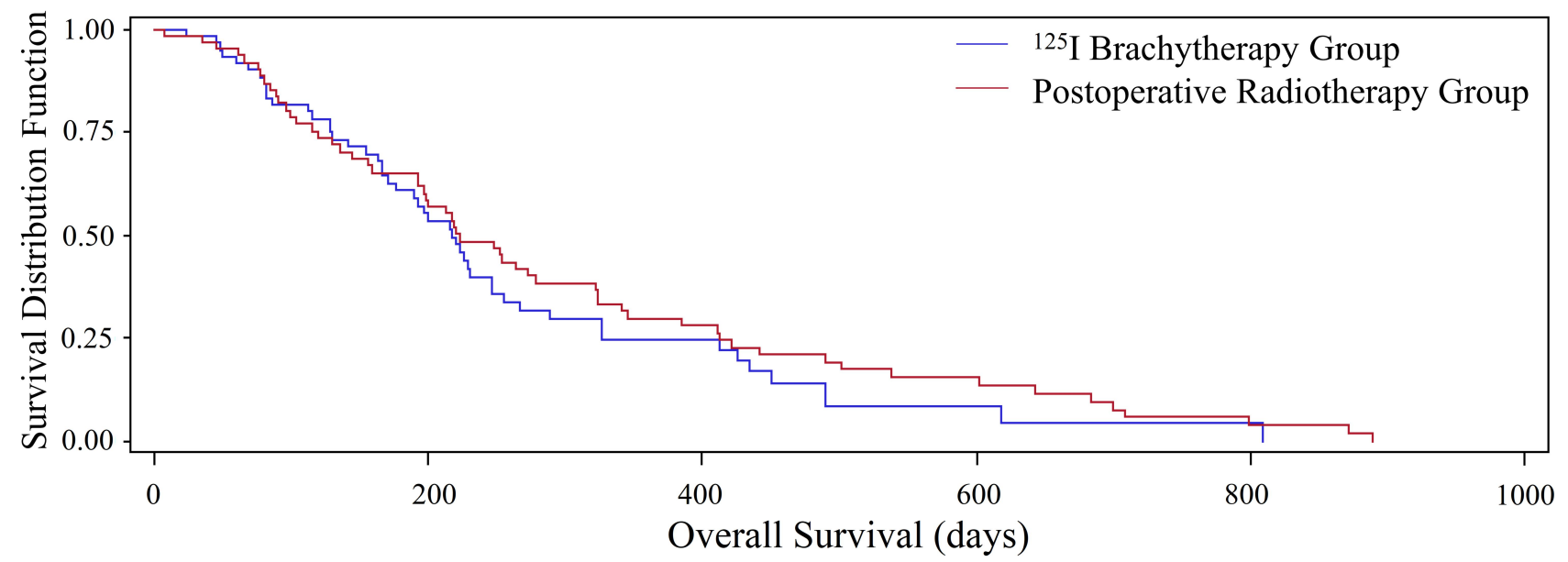

Figure 5 The postoperative overall survival curves for the two groups $(P=0.37$, Log rank test).

decompression and pedicle stabilization combined with postoperative radiotherapy. Although ${ }^{125} \mathrm{I}$ brachytherapy required more operation time and suffer from more intraoperative blood loss, it could obtain relatively better pain relief and less radiation-related complications as compared with conventional radiotherapy in the treatment of MESCC patients. The overall survival outcome and length of hospitalization were similar. Notably, the study was the first article with a large sample investigating the efficacy and safety of surgical decompression and spine stabilization combined with ${ }^{125}$ I brachytherapy seed implantation in the treatment of MESCC patients.

Radioactive particle brachytherapy is a precision radiation therapy which already has a history of more than one hundred years. This modality was initially performed to treat patients with prostate cancer and obtained satisfactory efficacy. ${ }^{15}$ Brachytherapy resulted in an improved biochemical relapse-free survival as compared with external beam radiotherapy alone in prostate cancer patients, ${ }^{16}$ and less acute rectal toxicity and better prostate-specific antigen control. ${ }^{17}$ So far, this modality has also been used to treat various cancer types, namely, lung cancer, oral tongue cancer, and squamous cell anal carcinoma. ${ }^{18-20}$

${ }^{125} \mathrm{I}$ seed implantation is a booming method which yields encouraging clinical results in the treatment of spine metastasis in other studies. ${ }^{10-13}$ Yang et al ${ }^{10}$ compared the clinical efficacy between the combination of percutaneous vertebroplasty (PVP) and ${ }^{125} \mathrm{I}$ implantation and regular radiotherapy alone for the treatment of spinal osteoplastic metastasis. The combination method was superior to radiotherapy alone in terms of pain relief and patient's quality of life. One week later, the VAS in the combined group dropped from 8.73 to 3.73 , but the radiotherapy group did not show a significant difference, from 8.34 to 8.43 . After one month, 2.54 in the combined group versus 5.46 in the radiotherapy group. After six months, 
Table 3 Univariate and Multivariate Analyses of Preoperative Factors for Postoperative Overall Survival in MESCC Patients

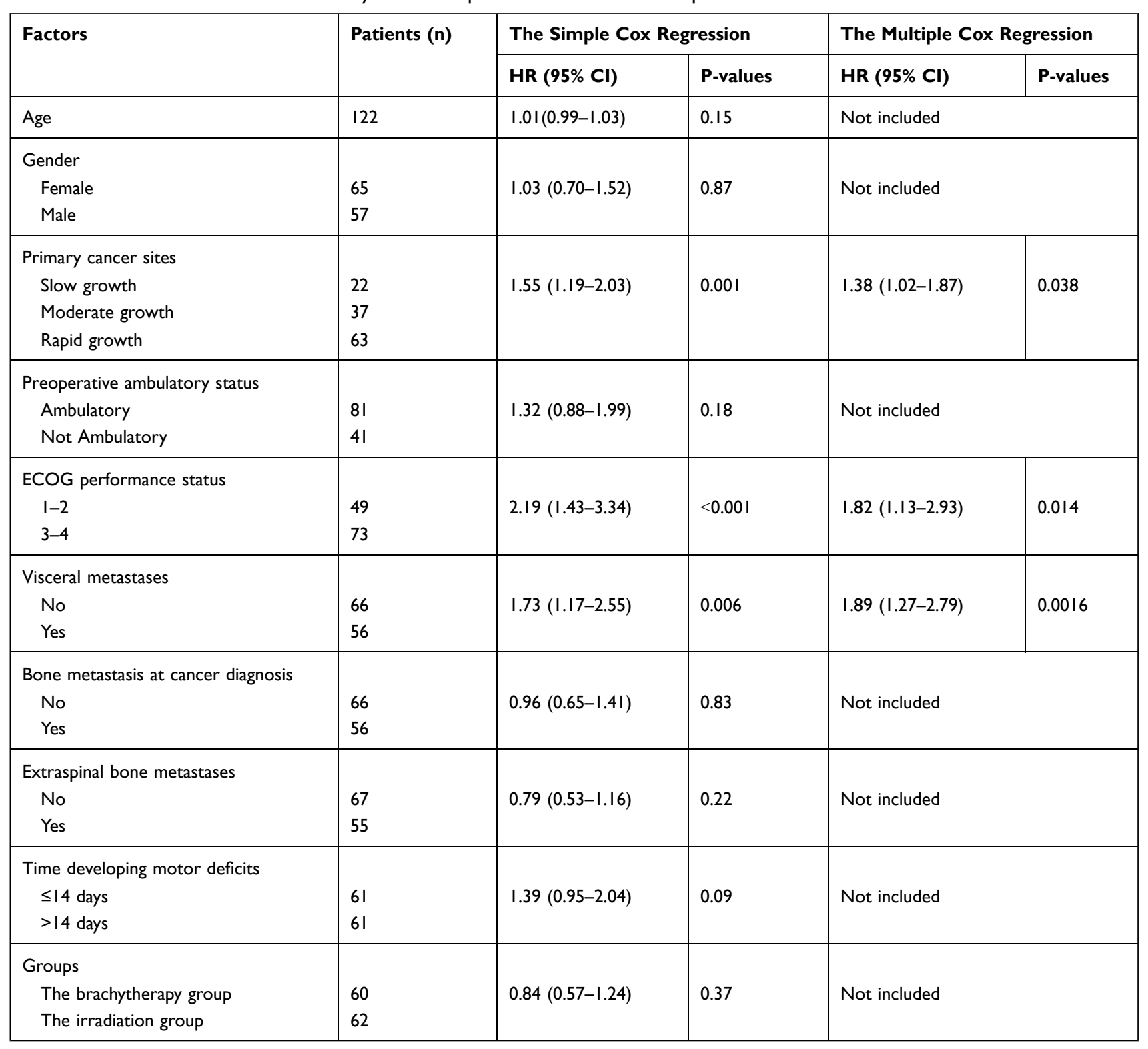

Abbreviations: MESCC, metastatic epidural spinal cord compression; ECOG, Eastern Cooperative Oncology Group; HR, hazard ratio; Cl, confidence interval.

1.25 versus 3.54. After 12 months, 1.32 versus 4.73. A similar decrease was shown in the score of life quality. $\mathrm{Li}$ et $\mathrm{al}^{11}$ found PVP combined with multi-needle implantation of ${ }^{125}$ I seeds in patients with osteolytic spinal metastasis appeared to be better than PVP combined with singleneedle implantation of ${ }^{125}$ I seeds in terms of clinical benefit rate and disease control rate because multi-needle implantation had greater bone cement injection volume and the more ideal distribution of the ${ }^{125} \mathrm{I}$ seeds. Decompressive surgery combining with precise ${ }^{125}$ I brachytherapy seed implantation facilitates rapid decompression, immediate metastatic radiation, and accurate tumor assessment, which has become a new modality for
MESCC patients. ${ }^{12,13}$ Rogers et $\mathrm{al}^{13}$ reported that surgical resection and ${ }^{125} \mathrm{I}$ brachytherapy was well tolerated and resulted in durable local control and ambulatory function outcome in patients with MESCC. Thirty patients were included in the study. The 2- and 3-year local control rate was $87.4 \%$ and $72.9 \%$, respectively. After surgery, $84 \%$ of patients had either normal or improve ambulatory status. The morbidity was restricted to 4 postoperative events. Qian et al ${ }^{12}$ concluded that pedicle fixation combined with ${ }^{125}$ I brachytherapy could effectively relieve short-term pain and improve patient's quality of life after retrospectively analyzing seven metastatic thoracolumbar tumor patients. However, the available studies were 
subject to small retrospectively samples, simple observations, and indistinct endpoints.

In the study, the postoperative VAS score was significantly lower compared with the preoperative scores in both groups, which suggested that both methods could significantly improve the patient's pain relief. Importantly, the VAS in the brachytherapy group was significantly lower than that in the irradiation group at 1 month, 3 months, and 6 months after surgery, which indicated that surgical decompression and pedicle stabilization combined with ${ }^{125}$ I brachytherapy had better pain relief as compared with surgical decompression and pedicle stabilization combined with postoperative radiotherapy. The ambulatory outcome was relatively better in the brachytherapy group, as compared with the patients in the irradiation group $(90.0 \%$ vs $83.9 \%, \mathrm{P}=0.32)$, but it did not reach the significance. Regarding survival prognosis, the median overall survival time was 7.27 months in the brachytherapy group and 7.43 months in the irradiation group, which showed no significance $(\mathrm{P}=0.37)$, and median overall survival time of 3 to 8 months was reported in other investigations.${ }^{3,14,21}$ Moreover, patients in the brachytherapy group were less likely to develop complications as compared with patients in the irradiation group ( $25.0 \%$ vs $46.8 \%, \mathrm{P}=0.0086)$. In detail, patients in the brachytherapy group had a lower rate of radiation-related complications as compared with patients in the irradiation group ( $8.3 \%$ vs $38.7 \%, \mathrm{P}<0.001)$. However, the rates of surgery-related complications and systematic complications were similar between the two groups $(\mathrm{P}=0.35$ and $\mathrm{P}=0.82$ ). These results were not surprised that the brachytherapy group had less radiation-related complications as compared with the irradiation group. Although the irradiation group had less operation time and intraoperative blood loss, the mean length of hospitalization length was similar in both groups. The above-mentioned results indicated surgical decompression and pedicle stabilization combined with ${ }^{125} \mathrm{I}$ brachytherapy was superior to surgical decompression and pedicle stabilization combined with postoperative radiotherapy in terms of pain relief and radiation-related complications. This method has the following advantages: (1) it could relieve the mechanical pain caused by metastatic compression and receive radiation therapy immediately after surgery; (2) the target dose was high enough to better kill biological pain caused by cancer cells; (3) the particles were targeted and matched to the metastatic lesion shape so it did not increase serious complications such as spinal cord injury.

According to the multiple Cox regression analysis, primary cancer sites, ECOG performance status, and visceral metastases were significant, which indicated that the three variables were the independent predictors for postoperative survival outcome. Bollen et $\mathrm{al}^{22}$ performed a systematic review with a focus on prognostic factors associating with survival in patients with spine metastasis. The authors concluded that the prognostic factors most frequently associated with survival were the primary tumor and performance status, and the prognostic factors most frequently not associated with survival were age, gender, number of spine metastases, location of the spine metastatic lesions, and the presence of a pathologic fracture after investigating 43 different prognostic factors.

We acknowledge surgical decompression and spine stabilization combined with ${ }^{125}$ I brachytherapy have some strengths and weaknesses. The strengths are as follows. First, this method can achieve adequate decompression, and radiation immediately after implanting radioactive seeds. In contrast, patients who are treated with decompressive surgery cannot receive regular radiotherapy until the surgical incision is healed, about 2 to 4 weeks after surgery. During this period, the local disease may lose control due to the progression of metastatic cancer. Second, the brachytherapy is conformal radiotherapy and can be precisely implanted. The extent of radiation can be matched to the metastatic tumor. The target dose is high, while the radiation dose of the surrounding normal soft-tissue is relatively low, thus enhancing the efficacy and decreasing the risk of radiationrelated complications, such as injury to the spinal cord. Third, precise ${ }^{125} \mathrm{I}$ brachytherapy seed can release ray for long periods, continuously inhibiting the growth of tumor cells and preventing the occurrence of local disease. Four, the half-life period of ${ }^{125}$ I is relatively short, which also makes it easy to protect their family members from radiation.

The weaknesses were as follows. For one thing, the halflife period of ${ }^{125} \mathrm{I}$ is 60.2 days, indicating that it spends about 60.2 days for the radioactive intensity decreasing to its half value. The radioactive effects on metastatic tumor lesions would also decrease with the reduction of the radioactivity. Thus, to realize better local control of disease, it is necessary to supplement the radioactivity with an additional method. For another thing, the indictor of ${ }^{125} \mathrm{I}$ dose is unclear. The excessive doses may result in severe radioactive damage, while insufficient doses cannot prevent the early occurrence of local disease. Therefore, further studies should investigate the relationship between the radioactive dose and corresponding effects. Besides, although this study had a relatively large sample, it was a retrospective study in a single center. Therefore, the external validation of the study needs further investigation. A prospective multicenter study with a larger sample was warranted. 


\section{Conclusion}

In conclusion, surgical decompression and spine stabilization combined with ${ }^{125}$ I brachytherapy is a relatively safe and useful method in MESCC patients.

\section{Funding}

This study was supported by the Medical and health science and technology innovation project of Sanya (NO. 2018YW04) and Beijing Municipal Science and Technology Commission (NO. Z161100000516101 and NO.Z171100001017176).

\section{Disclosure}

The authors report no conflicts of interest for this work.

\section{References}

1. Cole JS, Patchell RA. Metastatic epidural spinal cord compression. Lancet Neurol. 2008;7(5):459-466. doi:10.1016/S1474-4422(08) 70089-9

2. Lawton AJ, Lee KA, Cheville AL, et al. Assessment and management of patients with metastatic spinal cord compression: a multidisciplinary review. $J$ Clin Oncol. 2019;37(1):61-71. doi:10.1200/ JCO.2018.78.1211

3. Patchell RA, Tibbs PA, Regine WF, et al. Direct decompressive surgical resection in the treatment of spinal cord compression caused by metastatic cancer: a randomised trial. Lancet. 2005;366(9486):643648. doi:10.1016/S0140-6736(05)66954-1

4. Lee CH, Kwon JW, Lee J, et al. Direct decompressive surgery followed by radiotherapy versus radiotherapy alone for metastatic epidural spinal cord compression: a meta-analysis. Spine (Phila Pa 1976). 2014;39(9):E587-592. doi:10.1097/BRS.0000000000000258

5. Kim JM, Losina E, Bono CM, et al. Clinical outcome of metastatic spinal cord compression treated with surgical excision \pm radiation versus radiation therapy alone a systematic review of literature. Spine. 2012;37(1):78-84. doi:10.1097/BRS.0b013e318223b9b6

6. Keam J, Bilsky MH, Laufer I, et al. No association between excessive wound complications and preoperative high-dose, hypofractionated, image-guided radiation therapy for spine metastasis Clinical article. $J$ Neurosurg Spine. 2014;20(4):411-420. doi:10.3171/2013.12. SPINE12811

7. Sohn S, Chung CK, Sohn MJ, et al. Stereotactic radiosurgery compared with external radiation therapy as a primary treatment in spine metastasis from renal cell carcinoma: a multicenter, matched-pair study. J Neuro Oncol. 2014;119(1):121-128. doi:10.1007/s11060014-1455-9

8. Sohn S, Chung CK, Sohn MJ, Kim SH, Kim J, Park E. Radiosurgery compared with external radiation therapy as a primary treatment in spine metastasis from hepatocellular carcinoma: a multicenter, matched-pair study. J Korean Neurosurg Soc. 2016;59(1):37-43. doi:10.3340/jkns.2016.59.1.37
9. Haley ML, Gerszten PC, Heron DE, Chang YF, Atteberry DS, Burton SA. Efficacy and cost-effectiveness analysis of external beam and stereotactic body radiation therapy in the treatment of spine metastases: a matched-pair analysis. J Neurosurg Spine. 2011;14(4):537542. doi:10.3171/2010.12.SPINE10233

10. Yang ZZ, Tan J, Zhao RL, et al. Clinical investigations on the spinal osteoblastic metastasis treated by combination of percutaneous vertebroplasty and I-125 seeds implantation versus radiotherapy. Cancer Biother Radio. 2013;28(1):58-64.

11. Li T, Li J, Wang Z, Liu B, Han D, Wang P. A preliminary comparative clinical study of vertebroplasty with multineedle or single-needle interstitial implantation of (125)I seeds in the treatment of osteolytic metastatic vertebral tumors. J Neurosurg Spine. 2014;20(4):430-435. doi:10.3171/2014.1.SPINE13645

12. Qian J, Bao Z, Zou J, Yang H. Effect of pedicle fixation combined with (125)I seed implantation for metastatic thoracolumbar tumors. $J$ Pain Res. 2016;9:271-278.

13. Rogers CL, Theodore N, Dickman CA, et al. Surgery and permanent 125I seed paraspinal brachytherapy for malignant tumors with spinal cord compression. Int J Radiat Oncol Biol Phys. 2002;54(2):505513. doi:10.1016/S0360-3016(02)02961-9

14. Lei M, Li J, Liu Y, Jiang W, Liu S, Zhou S. Who are the best candidates for decompressive surgery and spine stabilization in patients with metastatic spinal cord compression? A new scoring system. Spine (Phila Pa 1976). 2016;41(18):1469-1476. doi:10. 1097/BRS.0000000000001538

15. Zaorsky NG, Harrison AS, Trabulsi EJ, et al. Evolution of advanced technologies in prostate cancer radiotherapy. Nat Rev Urol. 2013;10 (10):565-579. doi:10.1038/nrurol.2013.185

16. Hoskin PJ, Motohashi K, Bownes P, Bryant L, Ostler P. High dose rate brachytherapy in combination with external beam radiotherapy in the radical treatment of prostate cancer: initial results of a randomised phase three trial. Radiother Oncol. 2007;84(2):114-120. doi:10.1016/ j.radonc.2007.04.011

17. Deutsch I, Zelefsky MJ, Zhang Z, et al. Comparison of PSA relapsefree survival in patients treated with ultra-high-dose IMRT versus combination HDR brachytherapy and IMRT. Brachytherapy. 2010;9 (4):313-318. doi:10.1016/j.brachy.2010.02.196

18. Boukhelif W, Ferri-Molina M, Mazeron R, et al. Interstitial pulseddose-rate brachytherapy for the treatment of squamous cell anal carcinoma: a retrospective single institution analysis. Brachytherapy. 2015;14(4):549-553. doi:10.1016/j.brachy.2015.03. 006

19. Bansal A, Ghoshal S, Oinam AS, Sharma SC, Dhanireddy B, Kapoor R. High-dose-rate interstitial brachytherapy in early stage oral tongue cancer - 15 year experience from a tertiary care institute. $J$ Contemp Brachytherapy. 2016;1(1):56-65. doi:10.5114/jcb.2016.58082

20. Mutyala S, Stewart A, Khan AJ, et al. Permanent iodine-125 interstitial planar seed brachytherapy for close or positive margins for thoracic malignancies. Int J Radiat Oncol Biol Phys. 2010;76 (4):1114-1120. doi:10.1016/j.ijrobp.2009.02.067

21. Arrigo RT, Kalanithi P, Cheng I, et al. Predictors of survival after surgical treatment of spinal metastasis. Neurosurgery. 2011;68 (3):674-681; discussion 681. doi:10.1227/NEU.0b013e318207780c

22. Bollen L, Jacobs WCH, Van DL, et al. A systematic review of prognostic factors predicting survival in patients with spinal bone metastases. Eur Spine J. 2018;27:799-805. doi:10.1007/s00586-017$5320-3$ 


\section{Publish your work in this journal}

The Journal of Multidisciplinary Healthcare is an international, peerreviewed open-access journal that aims to represent and publish research in healthcare areas delivered by practitioners of different disciplines. This includes studies and reviews conducted by multidisciplinary teams as well as research which evaluates the results or conduct of such teams or healthcare processes in general. The journal covers a very wide range of areas and welcomes submissions from practitioners at all levels, from all over the world. The manuscript management system is completely online and includes a very quick and fair peer-review system. Visit http://www.dovepress.com/testimonials. php to read real quotes from published authors. 